\title{
A Compact 6-DoF Nonlinear Wave Energy Device Model for Power Assessment and Control Investigations
}

\author{
Giuseppe Giorgi, and John V. Ringwood, Senior Member, IEEE
}

\begin{abstract}
High accuracy at a low computational time is likely to be a fundamental trait for mathematical models for wave energy converters, in order to be effective tools for reliable motion prediction and power production assessment, device and controller design, and loads estimation. Wave energy converters are particularly prone to exhibit complex and nonlinear behaviours, which are difficult to be modelled efficiently. Highlynonlinear effects, related to nonlinear Froude-Krylov forces, are nonlinear coupling, instability, and parametric resonance, which may damage or improve the power production. It is therefore fundamental to be able to describe such nonlinearities, in order to assess their repercussion on the performance of the device, and eventually design the system in order to exploit them.

This paper provides a computationally efficient, compact, and flexible modelling approach for describing nonlinear FroudeKrylov forces for axisymmetric wave energy devices, in 6 degrees of freedom. Unlike other similar models, based on a mesh discretization of the geometry, the analytical formulation of the wetted surface allows the dynamical model to run almost in real time.
\end{abstract}

Index Terms-Nonlinear hydrodynamic model, parametric resonance, pitching/rolling instability, computationally efficient, wave energy converter.

\section{INTRODUCTION}

$\mathbf{W}$ AVE energy converters (WECs), by their nature, are prone to show significant and various nonlinearities, since their principal aim, pursued by the control strategy, is to enlarge the amplitude of motion, in order to maximize the absorbed power [1]. Consequently, linear models, although popular for their computational convenience, may, in certain conditions, return poor results, in term of accuracy of motion prediction and, therefore, power production assessment [2]. Furthermore, since power-optimizing control strategies may be really sensitive to modelling errors [3], using linear models as a base for the controller may be highly penalizing for the final power output.

The most important hydrodynamic nonlinearities in waveactivated WECs are due to large changes in the instantaneous wetted surface, which can be described by nonlinear FroudeKrylov (FK) force models [2]. Side effects of nonlinear FK forces are, eventually, nonlinear coupling between different

Manuscript received MONTH DAY, YEAR; revised MONTH DAY, YEAR; accepted MONTH DAY, YEAR. This paper was supported by Science Foundation Ireland, under Grant. no. 13/IA/1886. Paper no. NUMBER.

G. Giorgi and J. V. Ringwood are with the Centre for Ocean Energy Research (COER), National University of Ireland Maynooth, Co. Kildare, Ireland (e-mail: giuseppe.giorgi.2015@mumail.ie; john.ringwood@mu.ie). degrees of freedom (DoFs), and parametric resonance [4] [6]. Such highly-nonlinear effects are potentially damaging the efficiency of the device, since they open a channel for the energy to flow from the DoF where the power is harvested, to other un-exploited DoF. Effectively, the power is spilled, in order to feed into parasitic motions. Conversely, it may also happen that, due to nonlinear variations of the system parameters, conditions for instability are met, which may magnify the amplitude of motion in one or more DoFs. Eventually, if such magnification of motion is partially transmitted to the DoF where the energy is harnessed, the power produced would increase [7].

Such deleterious/beneficial phenomena are completely undescribed by linear theory which, therefore, is likely to wrongly estimate the power absorbed. Similarly, nonlinear models with a lower number of DoFs may neglect important energy exchange with unmodelled DoFs. For example, considering heaving devices subject to two dimensional waves, the roll DoF is usually not modelled, since it is not externally excited, therefore theoretically null motion is expected. However, if, under certain conditions, parametric roll kicks in, the roll DoF is internally excited, causing the energy to be suck from other DoFs and, consequently, implying power loss.

Models able to describe such nonlinear behaviours are generally computationally expensive because, for geometries of arbitrary complexity, a mesh-approach is required. For example, using the software LAMSWEC (Large Amplitude Motion Simulation of Wave Energy Converters) [8], supported by tank experiment evidence, [5] and [4] show that the SEAREV and Wavebob devices, respectively, are incline to parametric roll, with consequent significant power loss.

Despite its accuracy, LAMSWEC (and similar mesh-based approaches) are too slow to be considered viable for control applications, or for long term power production assessment. However, if the geometry is assumed to be axisymmetric, computationally efficient approaches are available, relying on the ability of describing analytically the whole wetted surface, thereby avoiding the use of a mesh [9], [10]. Note that such assumption is not particularly restrictive for the common WEC class of point absorbers, which are designed to be nondirectional, therefore axisymmetric.

The aim and novelty of this paper is to provide a mesh-free compact and computationally efficient $6-\mathrm{DoF}$ nonlinear hydrodynamic model, based on nonlinear FK force calculations. The CorPower device [11] is then considered as a case study, investigating if and when pitching instability and parametric 
resonance occur, and the consequent implication on the power produced by the device. In order to evaluate the advantages brought by the nonlinear model, results are compared with standard linear FK models. Furthermore, nonlinear models in both one, three, and six DoFs are considered, in order to discuss the significance of nonlinear coupling and parasitic motions, which may affect the energy production.

The reminder of the paper is organized as follows: Sect. II presents the theoretical background about the linear and nonlinear FK force calculations, as well as a brief description of the parametric resonance phenomenon. Section III presents the considered case study, and results are discussed in Sect. IV. Some final remarks and conclusions are given in $\mathrm{V}$.

\section{THEORETICAL BACKGROUND}

The basics of linear and nonlinear FK force calculations are proposed in Sect. II-A. A brief description of parametric resonance is given in II-B.

\section{A. Froude-Krylov forces}

In the framework of linear potential theory, FK forces correspond to the integral of the pressure of the undisturbed wave field over the wetted surface of the device. Such a pressure is defined, according to linear Airy's theory, as:

$$
p(x, z, t)=p_{s t}+p_{d y}=-\gamma z+\gamma \frac{\cosh (\chi(z+h))}{\cosh (\chi h)} \eta(x, t)
$$

where $p_{s t}=-\gamma z$ is the static pressure, $p_{d y}$ the dynamic pressure, $\gamma$ the specific weight of the sea water, $\eta(x, t)$ a 2 dimensional wave with amplitude $a$ and wave frequency $\omega$, $\chi$ the wave number, $h$ the water depth (defined according to a right-handed inertial frame of reference $(x, y, z)$, with the origin at the still water level (SWL)), $x$ pointing in the direction of propagation of the wave, and $z$ pointing upwards. It is then convenient to apply Wheeler's stretching to (1), as shown in [12]. Note that, in irregular sea conditions, the pressure formulation in (1) is used for each harmonic component of the wave spectrum in order to define the total pressure field. The accuracy of the pressure representation, and the effectiveness of the nonlinear FK force calculation is discussed in [12], for regular and irregular sea states, also considering nonlinear wave models, such as Rienecker-Fenton, for regular waves, and higher-order spectral (HOS) method for irregular waves, concluding that the use of Wheeler's stretching allows for sufficiently accurate computation of nonlinear FK force, even with nonlinear waves.

Froude-Krylov forces are computed by integrating the pressure, shown in equation (1), over the instantaneous wetted surface $S(t)$. In particular, static and dynamic FK force components can be defined, respectively, as follows:

$$
\begin{aligned}
\mathbf{F}_{F K_{s t}} & =\mathbf{F}_{g}+\iint_{S(t)}-\gamma z \mathbf{n} d S \\
\mathbf{F}_{F K_{d y}} & =\iint_{S(t)} p_{d y} \mathbf{n} d S
\end{aligned}
$$

where $\mathbf{n}=\left(n_{x}, n_{y}, n_{z}\right)$ is the unit vector normal to the surface, pointing outwards, and $\mathbf{F}_{g}$ is the gravity force. Likewise, FK torques are defined as follows:

$$
\begin{aligned}
\mathbf{T}_{F K_{s t}} & =\mathbf{r} \times \mathbf{F}_{g}+\iint_{S(t)}-\gamma z \mathbf{r} \times \mathbf{n} d S \\
\mathbf{T}_{F K_{d y}} & =\iint_{S(t)} p_{d y} \mathbf{r} \times \mathbf{n} d S
\end{aligned}
$$

where $\mathbf{r}$ is the position vector, and $\times$ is the cross product.

In the linear approximation, it is assumed that the wave steepness and the relative displacement between the wave and the device is small (wave-follower behaviour). Consequently, integrals (2a) to (3b) are defined with respect the -constantwetted surface at rest, and usually computed with boundary element method (BEM) solvers, like WAMIT [13]. Conversely, nonlinear FK forces are computed with respect to the instantaneous wetted surface, therefore taking the real position of the device, with respect to $\eta$, into account.

For a geometry of arbitrary complexity, the surface can be discretized through a mesh, computing the contribution to the force over each mesh panel [8]. Such an approach, though feasible, is computationally expensive, due to the recalculation, at each time step, of the instantaneous wetted surface, and consequent remeshing of the geometry. For axisymmetric buoys, a convenient parametrization of the wetted surface can ease the calculation of the FK integrals. In terms of validation, the method proposed in this paper, in its $1-\mathrm{DoF}$ version, is effectively compared with CFD simulations in [2], and shown to be more accurate than the linear model. Also, the present method is a computationally efficient version, although with a narrower range of applicability, of the LAMSWEC approach; when LAMSWEC is applied to axisymmetric geometries, it returns the same results of the proposed approach, as shown in [9] and [2]. Therefore, since LAMSWEC has been widely validated through wave tank tests (for the SEAREV device in [4], and the Wavebob device in [5]), and since the proposed approach provides results virtually identical to LAMSWEC (for axisymmetric devices), by extension there is high confidence on the accuracy of the results given by the present modelling approach.

The assumption of axisymmetric geometry allows the analytical description of the whole wetted surface. It is convenient to use an auxiliary right-handed frame of reference $(\hat{x}, \hat{y}, \hat{z})$, fixed on the body, with the origin at its centre of gravity $(C o G)$, and with the axis parallel to the inertial frame's axis when the body is at rest, the $\hat{z}$-axis pointing upwards. Such a frame is advantageous both because it makes the definition of nonlinear FK forces easier (therefore faster to compute), and because it implies constant and minimal inertial matrix, which simplifies the dynamic response simulation [14], [10].

The geometry of a generic buoy, symmetric around a vertical axis, can be described in cylindrical coordinates, with respect to the body-frame, as follows: 


$$
\left\{\begin{array}{l}
\hat{x}(\rho, \theta)=f(\rho) \cos \theta \\
\hat{y}(\rho, \theta)=f(\rho) \sin \theta \quad, \quad \theta \in[-\pi, \pi) \wedge \rho \in\left[\rho_{1}, \rho_{2}\right] \\
\hat{z}(\rho, \theta)=\rho
\end{array}\right.
$$

where $f(\rho)$ is a generic function of the vertical coordinate $\rho$, describing the profile of revolution of the axisymmetric body, as shown in [2].

Defining the FK forces in the body-frame requires to map the pressure from the inertial-frame (where it is defined) onto the surface of the body. The transformation, from $(x, y, z)$ to $(\hat{x}, \hat{y}, \hat{z})$, is represented by the Euler angle triad $(\varepsilon, \delta, \psi)$, corresponding to roll, pitch, and yaw angles, respectively. The 3-2-1 Euler angle sequence is the rotation convention commonly used for marine vehicles, thought as three sets of rigid rotations [15]:

$$
\left(\begin{array}{l}
\hat{x} \\
\hat{y} \\
\hat{z}
\end{array}\right)=\left[\begin{array}{ccc}
c \psi & -s \psi & 0 \\
s \psi & c \psi & 0 \\
0 & 0 & 1
\end{array}\right]\left[\begin{array}{ccc}
c \delta & 0 & s \delta \\
0 & 1 & 0 \\
-s \delta & 0 & c \delta
\end{array}\right]\left[\begin{array}{ccc}
1 & 0 & 0 \\
0 & c \varepsilon & -s \varepsilon \\
0 & s \varepsilon & c \varepsilon
\end{array}\right]\left(\begin{array}{l}
x \\
y \\
z
\end{array}\right)
$$

where the operands $c$ and $s$ stand for cosine and sine, respectively.

The change of coordinates, from Cartesian $(\hat{x}, \hat{y}, \hat{z})$ to cylindrical $(\rho, \theta)$, shown in (4), requires the inclusion of $\left\|\mathbf{e}_{\rho} \times \mathbf{e}_{\theta}\right\|$ in the integral, where $\mathbf{e}_{\rho}$ and $\mathbf{e}_{\theta}$ are unity vectors in the $\rho$ and $\theta$ directions, respectively. Furthermore, $\mathbf{n}$ can be expressed as $\frac{\mathbf{e}_{\rho} \times \mathbf{e}_{\theta}}{\left\|\mathbf{e}_{\rho} \times \mathbf{e}_{\theta}\right\|}$. Simplifying the denominator of $\mathbf{n}$, it follows that the integral in $(2 \mathrm{~b})$, for example, becomes:

$$
\mathbf{F}_{F K_{d y}}=\iint_{S(t)} p_{d y}(\hat{x}, \hat{y}, \hat{z}) \mathbf{n} d S=\int_{\theta_{1}}^{\theta_{2} \rho_{1}} \int_{d y}(\rho, \theta)\left(\mathbf{e}_{\rho} \times \mathbf{e}_{\theta}\right) d \rho d \theta
$$

Although such an approach is applicable to any geometry with revolution symmetry, the vast majority of axisymmetric point absorbers can be described as a combination of cylinders, cones, and spheres. However, note that discs (lids), which close the surface of a cylinder, cannot be described using cylindrical coordinates. To this end, polar coordinates are valid alternatives, much alike to the cylindrical one, shown in [14], [10].

Finally, the FK force integrals must be solved numerically using, for example, a trapezoidal rule. The computation time depends on the integration scheme utilized, a 2D-quadrature scheme [16], and on the relative and absolute tolerances used to approximate the integral, which have been set to $10^{-2}$ and $10^{0}$. The ultimate value of the computation time depends on hardware capabilities (Intel(R) Xeon(R) CPU (E5-1620 v3 @ $3.50 \mathrm{GHz})$ processor, with $16.0 \mathrm{~GB}$ RAM and Windows 7 Professional 64 bit), and on the complexity of the geometry. Indeed, real WEC buoys may be a combination of different sections, typically cylinders and cones, with each section of the buoy requiring an individual integral. For the CorPower geometry, which is described in Sect. III, it is found that the calculation time of the nonlinear FK forces, in 6-DoFs, is of the order $5 \cdot 10^{-2}$. The consequent run time for computing the response of the device to incoming wave is about 1.2 times the simulation time, effectively allowing the model to run almost in real time. However, it is important to point out that all calculations are performed in Matlab, which is between one and two orders of magnitude slower than lower level coding languages, such as $\mathrm{C}$ or Fortran [17]. Nevertheless, although the mesh-based LAMSWEC nonlinear FK model is coded in Fortran (which is a significantly faster implementation than Matlab), it has a run time about 10 times longer than the simulation time [8], therefore about one order of magnitude slower than the method proposed in this paper.

\section{B. Parametric resonance}

Parametric resonance is caused by time-varying changes in the parameters of a system [18]. Such a phenomenon is observed in floating bodies, when the roll natural period is twice the heave/pitch natural period, and it has been widely investigated in shipping and offshore spar-type structure.

However, little investigation has been undertaken in the wave energy field [19]. Numerical and experimental studies are available for the pitching device SEAREV [5], the 2-body self-reacting heaving device Wavebob [4], and a floating spartype oscillating water column device [6]. Such studies show detrimental effect of parametric motion to the WEC performance, since the energy is parametrically transferred from the primary mode of motion into other modes. Nevertheless, it is not excluded that parametric resonance may be beneficial for certain WECs, expressly designs to take advantage out of parametric excitations [7], [20].

In systems with time varying parameters, two conditions for the generation of parametric motion are defined by [7]: 1) the frequency of the excitation is approximately twice the natural frequency of the secondary system (frequency condition), and 2 ) the amplitude of the parametric excitation is larger than the damping of the secondary system (threshold condition). For example, the Wavebob device, which has time varying hydrostatic stiffness and position of the centre of mass, is particularly prone to parametric resonance, since its large roll natural period (about 20s) is parametrically excited by waves of 10 s period.

In general, the classic approach to investigate the likelihood of parametric roll is the Mathieu equation [18], which is a singe DoF unforced equation, with harmonic variations of the stiffness term. Although it can give some insight, it cannot forecast the severity of the eventual parametric response. Furthermore, several approximations are needed to reduce the full nonlinear 6-DoF model into a 1-DoF partially linearized model, which would fit into the Mathieu equation structure. In particular, linear and nonlinear coupling effects between the 6-DoFs are be neglected, and the variations of the stiffness term are assumed to be harmonic, which is not necessary the case. Finally, nonlinear variations of the excitation force are be neglected as well.

\section{CASE STUdY}

The 6-DoF nonlinear hydrodynamic model is used to simulate the response of a device to incoming waves. Regular 
waves are considered, in order to be able to study the effect of the wave height $\left(H_{w}\right)$ and wave period $\left(T_{w}\right)$, independently. Furthermore, since parametric resonance is particularly sensitive to the frequency and amplitude of the external excitation, as explained in Sect. II-B, it is convenient to study, at least at first, only monochromatic waves. A wide range of wave conditions, based on [21], is considered, with $T_{w} \in[4,15] \mathrm{s}$, step $0.5 \mathrm{~s}$, and $H_{w} \in[0.5,4] \mathrm{m}$, step $0.5 \mathrm{~m}$. However, waves steeper than $2 \%$ (low period and large height) are excluded.

The case study is a heaving buoy WEC, inspired by the CorPower [11] device, whose shape and dimension, based on [21], are shown in Fig. 1. Such a device presents the peculiarity of a pre-tensioning system, used to reduce the weight of the buoy, and the WaveSpring mechanism, which effectively acts as a negative spring [21].

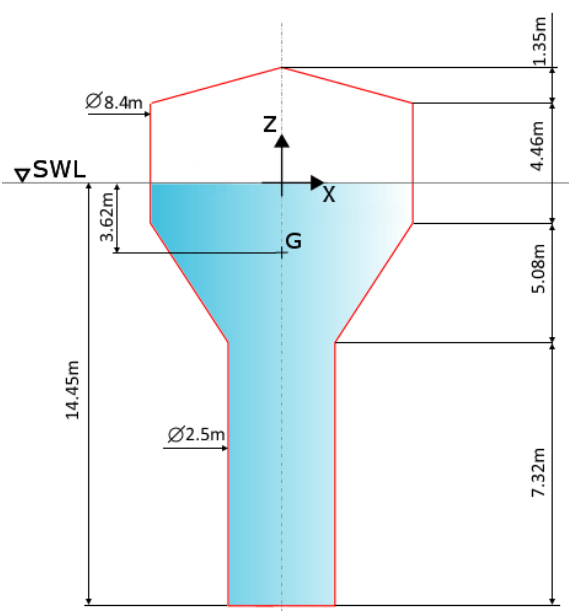

Fig. 1. Shape and dimensions of the case study buoy, inspired by the CorPower device [21].

The motion of the device is described by Newton's second law, in vectorial form, for 6 DoFs, written in the body-fixed frame of reference:

$$
\begin{aligned}
M \ddot{\mathbf{x}}= & \mathbf{f}_{F K_{s t}}+\mathbf{f}_{F K_{d y}}+\mathbf{f}_{d}+\mathbf{f}_{r a d}+\mathbf{f}_{v i s}+ \\
& \mathbf{f}_{C o r}+\mathbf{f}_{\text {moor }}+\mathbf{f}_{\text {pre }}+\mathbf{f}_{W S}+\mathbf{f}_{P T O},
\end{aligned}
$$

where $M$ is the inertial matrix, $\mathbf{x}=(\hat{x}, \hat{y}, \hat{z}, \varepsilon, \delta, \psi)$ is the state vector in the body-fixed frame, $\mathbf{f}$ is the generalized force vector, composed of 3 forces and 3 torques. The other force components in (7) are $\mathbf{f}_{d}$, the diffraction force, $\mathbf{f}_{r a d}$, the radiation force, $\mathbf{f}_{v i s}$, the viscous force, $\mathbf{f}_{C o r}$, the Coriolis force, $\mathbf{f}_{\text {moor }}$, the mooring force, $\mathbf{f}_{\text {pre }}$ the pretension force, $\mathbf{f}_{W S}$ the WaveSpring force, and $\mathbf{f}_{P T O}$, the power take-off (PTO) force. Note that $\mathbf{f}_{p r e}, \mathbf{f}_{W S}$, and $\mathbf{f}_{P T O}$ act on the axis of the device, therefore along $\hat{z}$.

The common expression for $\mathbf{f}_{d}, \mathbf{f}_{r a d}, \mathbf{f}_{v i s}$, and $\mathbf{f}_{C o r}$ are used [15], where $\mathbf{f}_{d}$ is computed through the diffraction impulse response function, $\mathbf{f}_{\text {rad }}$ through a state space representation of the radiation convolution integral [22], and $\mathbf{f}_{v i s}$ through a Morison equation [23]. Furthermore, $\mathbf{f}_{\text {moor }}$ and $\mathbf{f}_{\text {pre }}$ are linearly proportional to displacement, while $\mathbf{f}_{P T O}$ is linearly proportional to velocity.

The important numerical parameter values used in the model are based on [14], [21], and summarized in Table I,
TABLE I

MODEL PARAMETERS, BASED ON [14], [21].

\begin{tabular}{l|lll} 
& Surge $/$ Sway & Heave & Pitch $/$ Roll \\
\hline$M$ & $7.513 \cdot 10^{5} \mathrm{~kg}$ & $7.513 \cdot 10^{5} \mathrm{~kg}$ & $2.36 \cdot 10^{6} \mathrm{kgm}^{2}$ \\
$K_{\text {moor }}$ & $7.2 \cdot 10^{4} \mathrm{~N} / \mathrm{m}$ & $2 \cdot 10^{4} \mathrm{~N} / \mathrm{m}$ & $9.5 \cdot 10^{5} \mathrm{Nm} / \mathrm{m}$ \\
$K_{\text {pre }}$ & - & $5.17 \cdot 10^{5} \mathrm{~N} / \mathrm{m}$ & - \\
$B_{P T O}$ & - & $2.44 \cdot 10^{6} \mathrm{Ns} / \mathrm{m}$ & - \\
$C_{d}$ & 0.35 & 0.35 & 1 \\
$T_{n}$ & $13 \mathrm{~s}$ & $6.5 \mathrm{~s}$ & $7.5 \mathrm{~s}$
\end{tabular}

where $K_{\text {moor }}$ is the mooring stiffness, $K_{\text {pre }}$ is the pretension stiffness, $B_{P T O}$ the PTO damping coefficient, $C_{d}$ the drag coefficients, and $T_{n}$ the natural periods. The Wavespring mechanism, which is composed of three pneumatic cylinders, is modelled as in [24], assuming an adiabatic compression of the working fluid in the chambers of the cylinders. It follows that $\mathbf{f}_{W S}$ is computed as:

$$
\mathbf{f}_{W S}=N_{c} p_{W S} A_{W S} \hat{z} \sqrt{L_{W S, 0}^{2}+\hat{z}^{2}},
$$

where $N_{c}$ is the number of cylinders (3), $A_{W S}$ the active area of the cylinder $\left(0.1489 \mathrm{~m}^{2}\right), L_{W S, 0}$ the initial length of the cylinder $(4 \mathrm{~m})$, and $p_{W S}$ is the pressure in the chambers, defined as

$$
p_{W S}=p_{W S, 0} V_{W S, 0}^{\gamma_{a}} / V_{W S}^{\gamma_{a}}
$$

where $V_{W S}$ is the volume of the cylinder chamber, $\gamma_{a}$ the constant adiabatic index (1.4), and the subscript 0 indicates initial conditions.

\section{RESULTS}

The pitch response of the device, comparing the linear and nonlinear 6-DoFs models, is shown in Fig. 2, considering, for clarity, only waves of height $2 \mathrm{~m}$. Similar results are found for waves of different heights, with increasing nonlinear effects as the wave becomes higher, therefore steeper and more energetic.

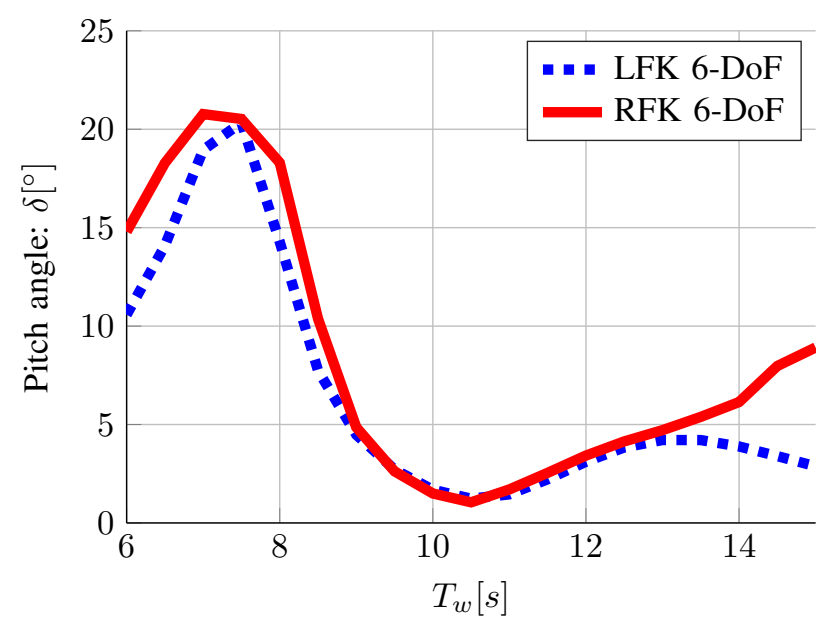

Fig. 2. Pitch response of the CorPower device, subject to regular waves of height $2 m$, according to linear and nonlinear Froude-Krylov 6-DoFs models.

Overall, the pitch response predicted by the nonlinear model is significantly larger, especially for periods close to the 
pitch resonant period (low periods), and close to the surge resonant period (high periods), which is coupled with pitch. The magnification of the pitch angle may have an effect on the power production since, as shown in [14], [10], the heave mode is nonlinearly coupled with surge and pitch modes.

The increase of the pitch response can be partially explained by the variations of the hydrostatic restoring torque in pitch, due to fluctuations of the metacentric height. In order to evaluate such variations, a parametric study is performed by displacing the buoy in fixed heave $\left(z_{G}\right)$ and pitch $(\delta)$ positions, and computing the hydrostatic torque $\left(T_{F K_{s t}}^{p}\right)$ over a wave cycle, considering, for example, a wave of $T_{w}=10 \mathrm{~s}$ and $H_{w}=2 m$.

Given the variability of nonlinear $T_{F K_{s t}}^{p}$, the mean torque is presented in Fig. 3, along with error bars, whose length is equal to the standard deviation of the static torque, over one wave period. While the nonlinear model significantly overlaps with the linear model for very small $z_{G}$, it diverges from it with when the absolute value of either $\delta$ or $z_{G}$ increases. Furthermore, for vertical displacements larger than $1.75 \mathrm{~m}$, the static torque changes sign, contriving to drive the buoy away from the equilibrium position, as opposed to acting as a restoring torque, which is the case for a linear model. In fact, for $z \geq 1.75 \mathrm{~m}$, the metacentre falls below the centre of gravity, which is the condition for pitching instability [25].

However, Fig. 3 only considers quasi-static variations of the system parameters, since the device is restrained from moving, and dynamic effects are nullified. Although quasistatic analysis permits a focus on $z_{G}$ and $\delta$ only, the dynamic response also needs to be considered, in order to take the effect of the natural frequency and the wave frequency into account, since they are important drivers of instability, as discussed in Sect. II-B, and shown in Fig. 4.

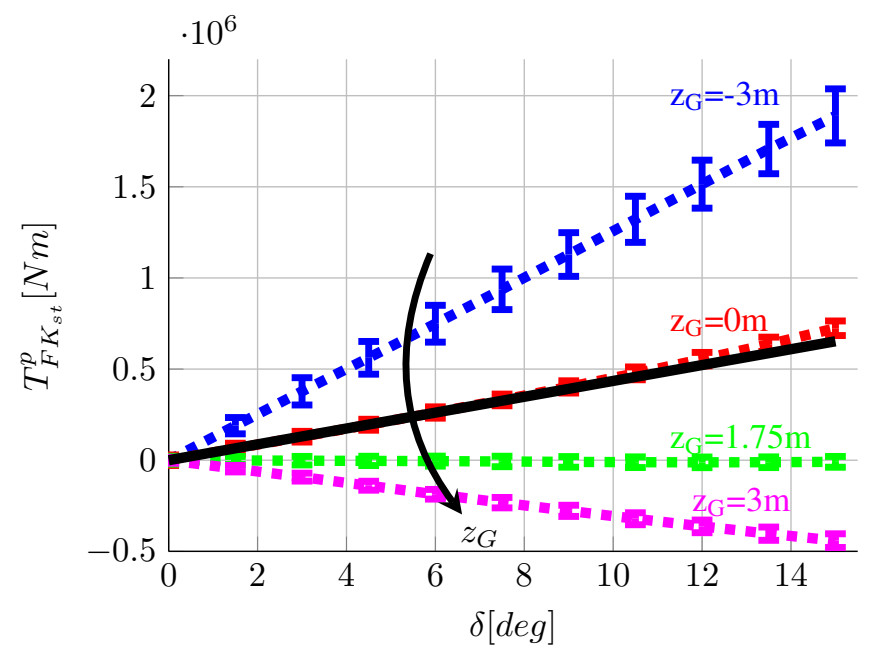

Fig. 3. Static pitch Froude-Krylov force $\left(T_{F K_{s t}}^{p}\right)$ for the CorPower buoy, subject to a wave with $H_{w}=2 m$ and $T_{w}=10 \mathrm{~s}$, according to the linear model (solid line), and the nonlinear model, for different heave position $z_{G}$.

The roll response is now considered, shown in Fig. 4. Note that the motion of the device is studied for a initial roll displacement of $0.1^{\circ}$, as suggested in [4], in order to provide some initial energy in the roll DoF. Since the external force is null in this DoF, the roll mode can be only internally excited, requiring some nonlinear mechanism for exchanging energy between different modes. Furthermore, in order to generate significant roll responses, parametric resonance must occur. In fact, when a system resonates, it magnifies negligible fluctuations from to the equilibrium position, to infinity if the system is un-damped, or until the damping limitations are met.

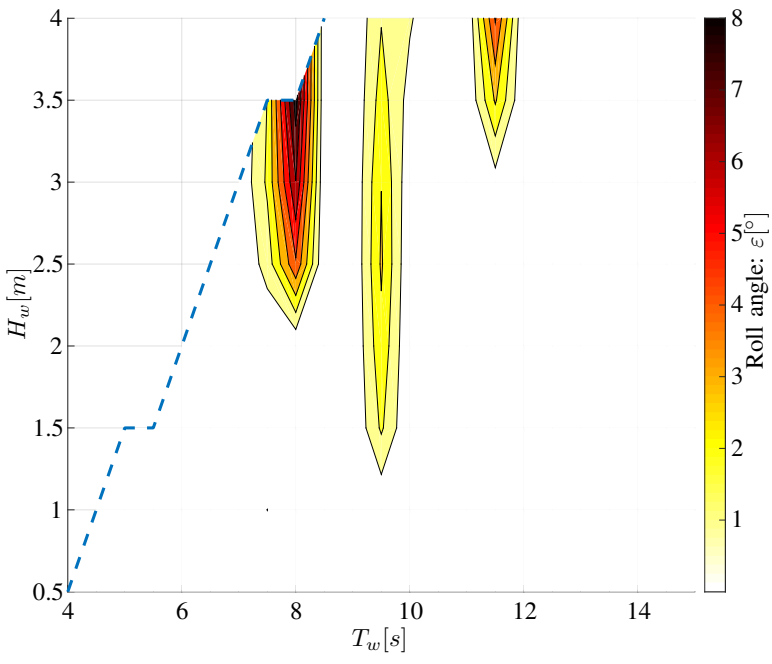

Fig. 4. Maximum roll angle, due to parametric resonance, according to the 6-DoF nonlinear Froude-Krylov model.

The conditions for parametric resonance act on the excitation amplitude and period, hence $H_{w}$ and $T_{w}$, respectively, as explained in II-B. Figure 4 clearly shows how parametric roll appears after a certain amplitude threshold is exceeded. The main periods where parametric roll is excited is at about $7.5-8 s$, where the maximum roll amplitude is about $8^{\circ}$. Smaller parametric roll responses are found at $11.5 \mathrm{~s}$ and $9.5 \mathrm{~s}$.

In other WECs with large roll natural period, for example [4] $\left(T_{n}\right.$ about 20s), parametric resonance happens at wave periods about half the roll natural period, therefore $T_{w}$ of about $10 \mathrm{~s}$. Conversely, for the CorPower device, the roll $T_{n}$ is quite small (about $7.5 \mathrm{~s}$ ), therefore waves with $T_{w}=\frac{1}{2} T_{n}$ are too small, and outside the target production region. However, since $T_{n}$ is small in roll, parametric resonance is indeed mainly happening at about $T_{w}=T_{n}$, as shown in Fig. 4 .

The response in roll, for the wave condition with largest roll amplitude $\left(T_{w}=8 s, H_{w}=3.5 \mathrm{~m}\right)$ is shown in the phase graph $(\varepsilon, \dot{\varepsilon})$, and fast Fourier transform (FFT), in Fig. 5. The frequency components of the FFT are normalized against the wave frequency $\omega_{w}$. The limit cycle response is quite simple, dominated by a relative frequency of unity. Such a response confirms that $T_{w}=8 s$ is the main wave frequency where parametric roll is directly excited. Furthermore, non-zero mean values and super-harmonics are evident.

Figure 4 shows that the secondary frequency where parametric roll is excited is at $T_{w}=11.5 \mathrm{~s}$. The phase diagram and FFT of the roll response to a wave of $T_{w}=11.5 \mathrm{~s}$ and $H_{w}=4 m$ is shown in Fig. 6. It can be noted that the limit cycle response is topologically more complex. Furthermore, the FFT graph shows that sub-harmonics are generated. The 

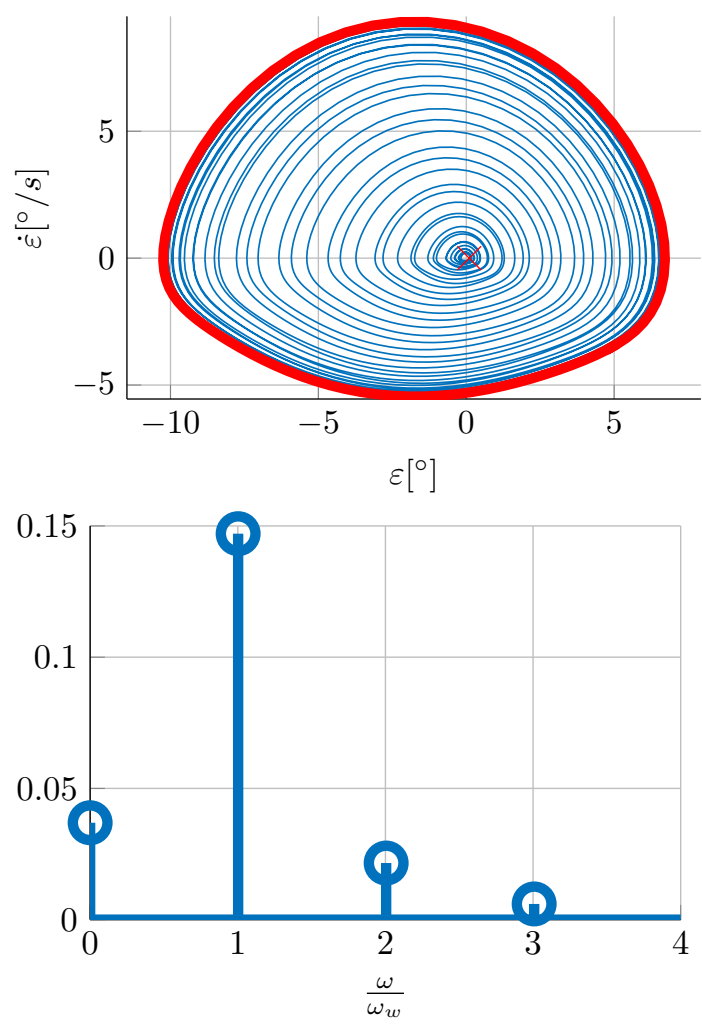

Fig. 5. Phase graph $(\varepsilon, \dot{\varepsilon})$ (top figure), and FFT (bottom figure), for the wave condition with $T_{w}=8 s$ and $H_{w}=3.5 \mathrm{~m}$. In the top figure, the cross indicates the initial condition $\left(\varepsilon=0.1^{\circ}, \dot{\varepsilon}=0^{\circ}\right)$, while the external solid line represents the limit cycle response.

dominant relative frequency is 1.5 , which corresponds to a period of $7.5 \mathrm{~s}$, which is exactly the roll natural period. Therefore, it can be inferred that parametric roll at $T_{w}=11.5 \mathrm{~s}$ is caused, in an indirect way, by the roll natural period.

Finally, the consequences of pitching instability and parametric roll on the energy absorption are investigated. In particular, neglecting one ore more DoF can affect the power production estimation. For the case of a heaving buoy, as the one studied in this paper, a 1-DoF linear model should predict the same power capture as a 6-DoF, since heave is linearly un-coupled from all the other modes. Conversely, nonlinear models take nonlinear coupling effects into account, therefore there could be differences between different models. In order to include, selectively, pitching nonlinearities, and parametric resonance, 1-(only heave), 3-(surge-heave-pitch), and 6-DoFs model are compared. Figure 7 shows the power production, estimated with such models.

Obviously, for small wave heights, the three models overlap, since nonlinear effects are minimal. For higher waves. the 3DoF model produces, overall, more power than the 1-DoF model, apart from wave periods close to $8 s$, where a significant power drop is found. Hence, it can be inferred that the power differences are due to the presence of the pitching motion, which is nonlinearly coupled with heave, as shown in [14], [10]. On the one hand, pitch angles up to about $15^{\circ}\left(T_{w}\right.$ greater than $9 s$, as shown in 2) benefit the power conversion. On the other hand, too large pitch angles seem to be detrimental $(\varepsilon$
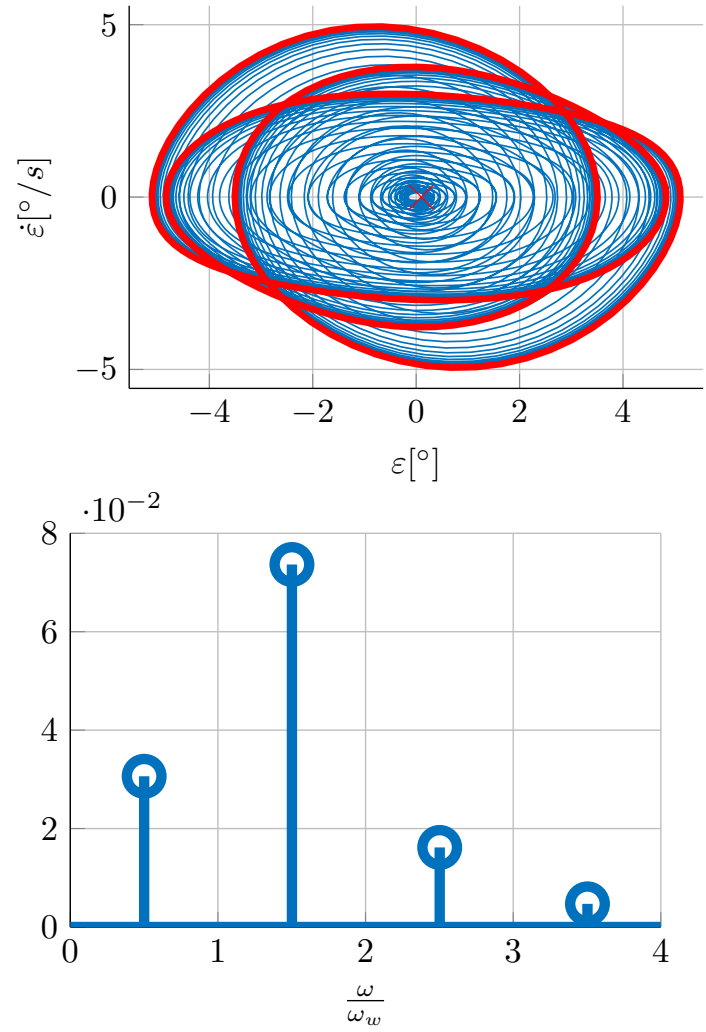

Fig. 6. Phase graph $(\varepsilon, \dot{\varepsilon})$ (top figure), and FFT (bottom figure), for the wave condition with $T_{w}=11.5 \mathrm{~s}$ and $H_{w}=4 \mathrm{~m}$. In the top figure, the cross indicates the initial condition $\left(\varepsilon=0.1^{\circ}, \dot{\varepsilon}=0^{\circ}\right)$, while the external solid line represents the limit cycle response.

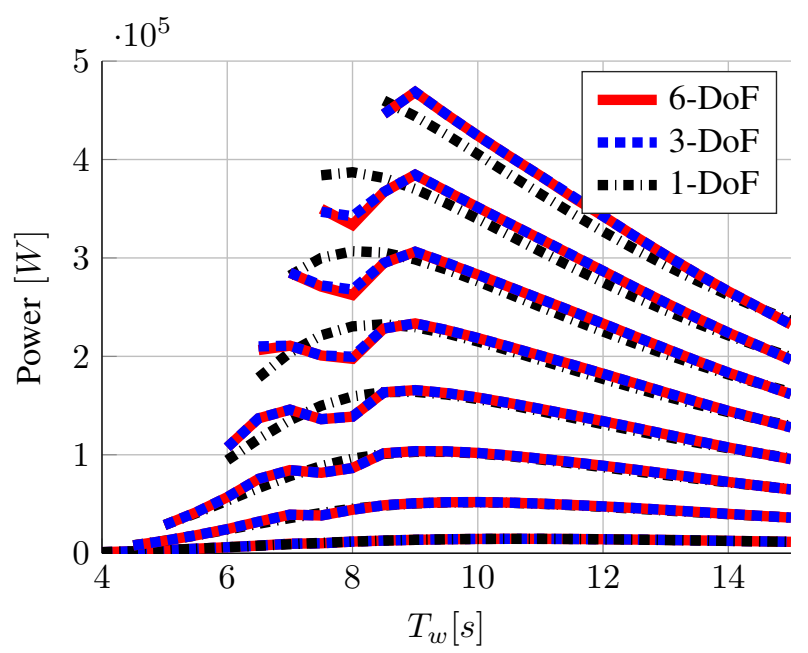

Fig. 7. Power estimation, according to the 1-, 3-, and 6-DoFs nonlinear Froude-Krylov models.

up to about $22^{\circ}$, at $T_{w}$ of about $7.5 \mathrm{~s}$, as shown in 2).

Small differences are found between the 3-DoFs and the 6DoFs models, indicating that, for the CorPower device, the influence of parametric roll to the power production is effectively negligible. Indeed, despite present, the parametric roll angles are relatively small. However, for the wave condition where the roll angle is the maximum $\left(8^{\circ}\right.$, at $T_{w}=8 s$, and $\left.H_{w}=3.5 \mathrm{~m}\right)$, the power difference, although small, is appreciable. 
In order to provide a clearer representation of the power production under- or over-estimation, Fig. 8 shows the ratio between the power estimated with the 6-DoF model, compared to the power estimated with the 1-DoF model. Consistent with Fig. 7, modest overestimation is found at large periods, with peaks of $+5 \%$. However, larger excursions are found at $T_{w}$ between $6 \mathrm{~s}$ and $9 \mathrm{~s}$, with up to $+16 \%$ overestimation at around $T_{w}=7 s$, and up to $-15 \%$ for $T_{w}=8 s$.

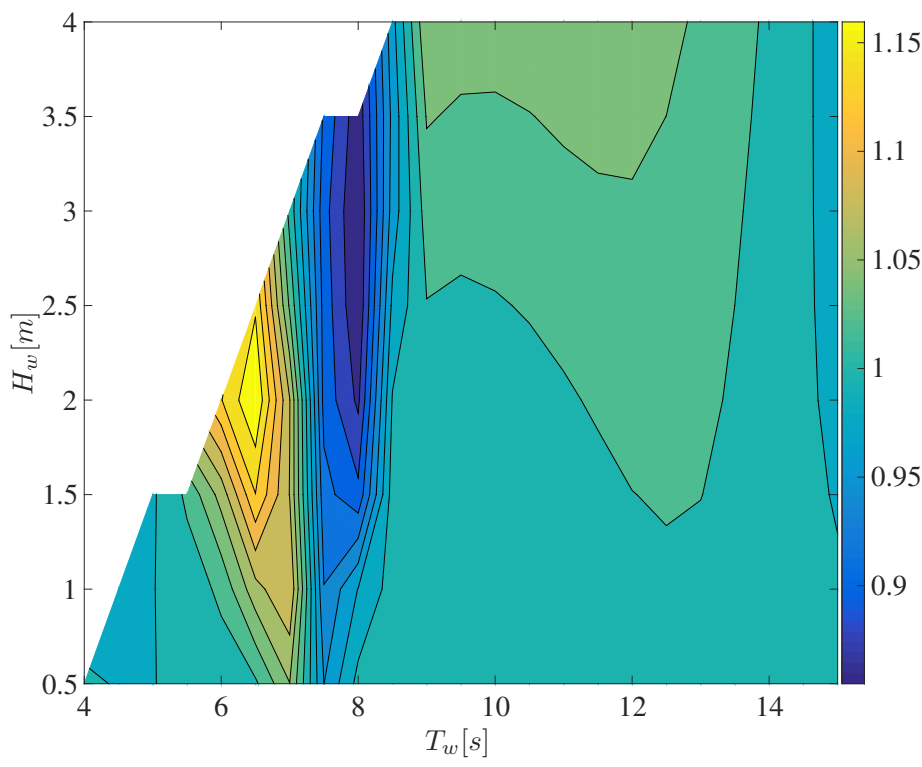

Fig. 8. Power production estimation ratio, between the 6-DoF and the 1-DoF model.

\section{CONCLUSION}

High accuracy at a low computational time is likely to be a fundamental trait for WECs mathematical models to be effective, in particular for reliable power production assessment, device and controller design, and loads estimation. Wave energy converters are particularly prone to exhibit complex and nonlinear behaviours, which are difficult to be modelled efficiently. Highly-nonlinear phenomena, often affecting the efficiency and the energy conversion capabilities of wave energy devices, are related to instabilities and parametrically excited motions.

This paper provides a computationally efficient, compact, and flexible modelling approach for describing nonlinear Froude-Krylov forces for axisymmetric wave energy devices, in 6 degrees of freedom. Unlike other similar models, based on a mesh discretization of the geometry, the analytical formulation of the wetted surface allows the dynamical model to run almost in real time. However, a considerable speed up, of about 1 or 2 order of magnitudes, is expected if lower level coding languages are employed.

The 6-DoF nonlinear model is used to investigate the response of the a having buoy wave energy converter, inspired by the CorPower device. The nonlinear model is shown to be able to represent pitching nonlinearities, and parametric resonance in the roll degree of freedom. In particular, nonlinear variations of the restoring pitch torque is discusse, with particular focus on instability conditions. Furthermore, the conditions for parametric roll response are discussed. Due to the relatively small roll natural period, comparable with the incoming wave period, two different parametric excitation mechanism are found: the first direct, at the roll natural period (with generation of super-harmonics), and the second indirect, at larger period (with generation of sub-harmonics).

Finally, the repercussion that such nonlinearities have on the power generation is investigated. In particular, 1-, 3-, and 6-DoFs model are considered, in order to progressively 15 study the influence of pitch and roll nonlinearities. It is found that, thanks to a nonlinear coupling between heave and pitch, the effect of pitch is usually beneficial, when the pitch angles are lower than $15^{\circ}$, while is detrimental for larger pitch angles, close to the pitch resonant period. On the other hand, parametric roll, although present, has no large effect on the power production since, when it happens, pitch roll are generally relatively small.

Overall, this paper provides a tool, easy to implement, to investigate nonlinear effects in wave energy converters, and their consequences on the efficiency of the device. As a future study, other devices, renown to show important parametric motions, and significantly affecting the power absorption, may be considered as a further case study. Moreover, as further work, a complete validation of the code is needed, using data from either CFD codes or experimental tests.

\section{ACKNOWLEDGMENT}

This paper is based upon work supported by Science Foundation Ireland under Grant No. 13/IA/1886.

\section{REFERENCES}

[1] M. Penalba, G. Giorgi, and J. V. Ringwood, "Mathematical modelling of wave energy converters: A review of nonlinear approaches," Renewable and Sustainable Energy Reviews, vol. 78, pp. 11881207, 2017. [Online]. Available: http://www.eeng.nuim.ie/coer/wpcontent/uploads/2017/05/J270MRGG-3.pdf

[2] G. Giorgi and J. V. Ringwood, "Froude-krylov and viscous drag representations in nonlinear wave energy devices models in the computation/fidelity continuum,' Ocean Engineering, vol. 141, pp. 164-175, 2017.

[3] F. Fusco and J. V. Ringwood, "Hierarchical robust control of oscillating wave energy converters with uncertain dynamics," IEEE Transactions on Sustainable Energy, vol. 5, no. 3, pp. 958-966, 2014.

[4] K. R. Tarrant, "Numerical modelling of paramtetric resonace of a heaving point absorber wave energy converter," Ph.D. dissertation, Department of Mechanical \& Manufacturing Engineering, Trinity College, March 2015.

[5] A. Babarit, H. Mouslim, A. Clément, and P. Laporte-Weywada, "On the numerical modelling of the non linear behaviour of a wave energy converter," in ASME 2009 28th International Conference on Ocean, Offshore and Arctic Engineering. American Society of Mechanical Engineers, 2009, pp. 1045-1053.

[6] R. Gomes, J. Malvar Ferreira, S. Ribeiro de Silva, J. Henriques, and L. Gato, "An experimental study on the reduction of the dynamic instability in the oscillating water column spar buoy," in 12th European Wave and Tidal Energy Conference (EWTEC), Cork, 2017.

[7] R. Galeazzi and K. Pettersen, Parametric resonance in dynamic systems, Controlling parametric resonance: induction and stabilization of unstable motions. Springer, 2012.

[8] J.-C. Gilloteaux, "Mouvements de grande amplitude d'un corps flottant en fluide parfait. application à la récupération de l'énergie des vagues." Ph.D. dissertation, Ecole Centrale de Nantes-ECN, 2007.

[9] G. Giorgi and J. V. Ringwood, "Computationally efficient nonlinear froude-krylov force calculations for heaving axisymmetric wave energy point absorbers," Journal of Ocean Engineering and Marine Energy, vol. 3, no. 1, pp. 21-33, 2017. 
[10] _ "Analytical formulation of nonlinear froude-krylov forces for pitching point absorbers," in 37th International Conference on Ocean, Offshore and Arctic Engineering, 2018.

[11] CorPower, "Corpower ocean ab, available at http://www.corpowerocean.com/," 2017.

[12] G. Giorgi and J. V. Ringwood, "Relevance of pressure field accuracy for nonlinear froude-krylov force calculations for wave energy devices," Journal of Ocean Engineering and Marine Energy, vol. 4, no. 1, pp. $57-71,2017$.

[13] W. Inc., WAMIT v7.0 manual, 2013.

[14] G. Giorgi and J. V. Ringwood, "Analytical representation of nonlinear froude-krylov forces for 3-dof point absorbing wave energy devices," submitted to Ocean Engineering, 2018.

[15] T. I. Fossen, Handbook of marine craft hydrodynamics and motion control. John Wiley \& Sons, 2011.

[16] L. Shampine, "Matlab program for quadrature in 2d," Applied Mathematics and Computation, vol. 202, no. 2008, pp. 266-274, 2008.

[17] F. F. Wendt, Y.-H. Yu, K. Nielsen, K. Ruehl, T. Bunnik, I. Touzon, B. W. Nam, J. S. Kim, K.-H. Kim, C. E. Janson et al., "International energy agency ocean energy systems task 10 wave energy converter modeling verification and validation," in 12th European Wave and Tidal Energy ConferencEuropean Wave and Tidal Energy Conference. Technical Committee of the European Wave and Tidal Energy Conference, 2017.

[18] T. Fossen and H. Nijmeijer, Parametric resonance in dynamical systems. Springer Science \& Business Media, 2011.

[19] J. Davidson, T. Kalmar-Nagy, G. Giorgi, and J. V. Ringwood, "Nonlinear rock and roll - modelling and control of parametric rresonances in wave energy devices," in Proceedings of the 9th Vienna International Conference on Mathematical Modelling, February 21-23, Vienna, Austria, 2018.

[20] B. Orazov, A novel excitation scheme for an ocean wave energy converter. University of California, Berkeley, 2011.

[21] J. H. Todalshaug, G. S. Asgeirsson, E. Hjálmarsson, J. Maillet, P. Möller, P. Pires, M. Guérinel, and M. Lopes, "Tank testing of an inherently phase-controlled wave energy converter," International Journal of Marine Energy, vol. 15, pp. 68-84, 2016.

[22] R. Taghipour, T. Perez, and T. Moan, "Hybrid frequency-time domain models for dynamic response analysis of marine structures," Ocean Engineering, vol. 35, no. 7, pp. 685-705, May 2007.

[23] G. Giorgi and J. V. Ringwood, "Consistency of viscous drag identification tests for wave energy applications," in 12th European Wave and Tidal Energy Conference (EWTEC), Cork, 2017.

[24] M. Guerinel, E. Jansson, J. H. Todalshaug, M. Jesmani, and K. Guijt, "Modelling alternatives for a heaving point absorber with and without stiffness modulation," in 12th European Wave and Tidal Energy Conference (EWTEC), Cork, 2017.

[25] A. Biran and R. L. Pulido, Ship hydrostatics and stability. ButterworthHeinemann, 2013

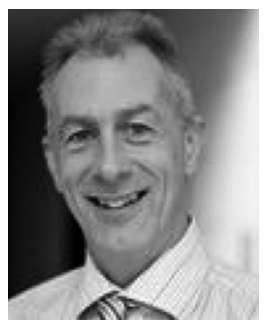

John V. Ringwood (M'87-SM'97) received the Diploma degree in electrical engineering from Dublin Institute of Technology, Dublin, Ireland, in 1981, and the Ph.D. degree in control of steel mills from Strathclyde University, Glasgow, U.K., in 1985. $\mathrm{He}$ is currently a Professor of electronic engineering at Maynooth University, Maynooth, Ireland. From 2000 to 2006, he was the Head of the Electronic Engineering Department, Maynooth University. His research interests include time series modelling, wave energy, control of plasma processes, and biomedical engineering. He is a Chartered Engineer and a Fellow of the Institution of Engineers of Ireland.

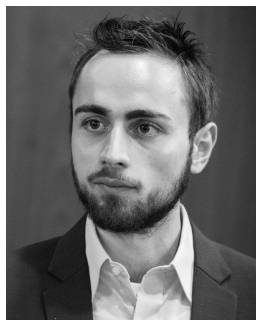

Giuseppe Giorgi received the Laura Magistrale in mechanical engineering from the Politecnico di Torino, Torino, Italy, in 2014. He is currently working toward the Ph.D. degree at the Centre for Ocean Energy Research, in the National University of Ireland Maynooth, Co. Kildare, Ireland. The topic of his study is nonlinear hydrodynamic modelling of wave energy converters, under controlled conditions, in order to create computationally efficient and accurate models for power production assessment and control applications. 